\title{
DISPERSION PROPERTIES OF ERGODIC TRANSLATIONS
}

\author{
STEFANO ISOLA
}

Received 19 November 2005; Revised 23 January 2006; Accepted 25 January 2006

We consider irrational rotations of the circle $T x=x+\alpha(\bmod 1)$ and study the asymptotic behavior of sums of the type $S_{n}=\sum_{k=0}^{n-1} \pi_{k}$ with $\pi_{k}= \pm 1$, the sign being determined by the location of $T^{k} x$ with respect to a binary partition.

Copyright (c) 2006 Hindawi Publishing Corporation. All rights reserved.

\section{Introduction and preliminaries}

Let $X=\mathbb{R} / \mathbb{Z}$ and $T: X \rightarrow X$ be the rotation of an angle $0<\alpha<1$, that is,

$$
T x=x+\alpha \quad(\bmod 1) .
$$

The Lebesgue measure $\mu$ is obviously $T$-invariant. The subset of $X$ given by $\{x+k \alpha\}_{k \in \mathbb{Z}}$ is the (full) orbit of $x$ under $T$. If $\alpha \in \mathbb{Q}$, say $\alpha=p / q$, then all orbits are finite (with $q$ points), whereas for $\alpha \in \mathbb{R} \backslash \mathbb{Q}$ all orbits are dense (the system is a minimal homeomorphism of $X$ ) and $\mu$ is the unique (and thus ergodic) invariant measure.

Let us consider the partition $\zeta=\left(A_{-}, A_{+}\right)$of $X$ into the half-open arcs

$$
A_{+}=\left\{x \in X: 0 \leq x<\frac{1}{2}\right\}, \quad A_{-}=\left\{x \in X: \frac{1}{2} \leq x<1\right\} .
$$

If the rotation angle $\alpha$ is irrational, then $\zeta$ is generating for $T$ (see [4]) and the partition $\zeta^{n}=\zeta \vee T \zeta \vee \cdots \vee T^{n-1} \zeta$ is made out of $2 n$ arcs. This can be easily realized by induction: when passing from $\zeta^{n-1}$ to $\zeta^{n}$ one has to add to the endpoints of the arcs belonging to $\zeta^{n-1}$ the two new points $T^{n}(0)$ and $T^{n}(1 / 2)$ (for rational $\alpha$, say $\alpha=p / q$, the partition $\zeta^{n}$ has precisely $2 q$ arcs for all $n \geq q$ ). Thus, the rotation is metrically isomorphic to the subshift given by the closure of $\pi([0,1))$ where the coding map $\pi:[0,1] \rightarrow\{-1,1\}^{\mathbb{Z}}$ can be defined by $\pi(x)_{n}=\vartheta\left(T^{n}(x)\right)$ and $\vartheta: X \rightarrow\{-1,1\}$ is the function in $L^{2}(X, \mu)$ given by

$$
\vartheta(x)=2 \chi_{A_{+}}(x)-1= \begin{cases}+1 & \text { if } x \in A_{+} \\ -1 & \text { if } x \in A_{-} .\end{cases}
$$

Hindawi Publishing Corporation International Journal of Mathematics and Mathematical Sciences Volume 2006, Article ID 20568, Pages 1-20

DOI 10.1155/IJMMS/2006/20568 
Here we want to study the asymptotic behavior of the ergodic sums

$$
S_{n}(x)=\sum_{k=0}^{n-1} \pi_{k}(x)
$$

Since

$$
\mu\left(S_{n}\right)=\int_{X} \sum_{k=0}^{n-1} \vartheta(x+k \alpha) \mu(d x)=n \int_{X} \vartheta(x) \mu(d x)=0,
$$

the faster $S_{n}$ grows to infinity the slower the convergence in the ergodic theorem is. The reason why we are considering this problem for the particular function $\vartheta$ is that we want to study a model of random walk on $\mathbb{Z}$ with transition to the closest neighbors when the medium is defined by the rotation $T: X \rightarrow X$. In this view $\left|S_{n}(x)\right|$ measures how far from the origin the walker is at the $n$th step. Due to the above observations on the partition $\zeta^{n}$ there are exactly $2 n$ different walks of length $n$ that can be produced in this way (for $\alpha$ irrational). If we introduce the involution $\sigma: X \rightarrow X$ which acts as $\sigma(x)=(x+1 / 2)(\bmod 1)$, then we plainly have $S_{k}=-S_{k} \circ \sigma$, whence the paths come in pairs symmetric with respect to the origin. Several mathematical problems arise in this context (for related investigations see $[1,15])$. The first is the characterization of the diffusive behavior of the walk, that is, the growth behavior of $S_{n}$. Other interesting items may arise in the attempt to understand the first passage distribution function

$$
f(n)=\mu\{x \in X: \tau(x)>n\}
$$

with

$$
\tau(x)=\min \left\{n: S_{n}(x)=0\right\},
$$

as well as the limit probability density

$$
p(t)=\lim _{n \rightarrow \infty} \mu\left\{x \in X: \tau_{n}^{ \pm}(x)=n t\right\}
$$

where $\tau_{n}^{ \pm}$is the time spent by the walker on the right (resp., left) side of the origin up to time $n$, that is,

$$
\tau_{n}^{ \pm}(x)=\frac{1}{2} \sum_{k=0}^{n-1}\left(1 \pm \operatorname{sgn} S_{k}(x)\right)
$$

Recall that for the ordinary symmetric random walk, where the diffusive behavior is a normal one, one finds $f(n)=O\left(n^{-1 / 2}\right)$ and $p(t)=1 / \pi \sqrt{t(1-t)}$.

In this paper only the first problem will be addressed, leaving the last two to further research. We mention in passing that this problem also arises in the attempt to understand the asymptotic behavior of the following skew product on the cylinder:

$$
(x, y) \longrightarrow(x+\alpha, y+\vartheta(x)) .
$$


The asymptotic behavior of the $y$-variable is completely determined by that of $S_{n}$. This has been investigated in [5] for a class of Liouville rotation angles. On the other hand, in [7] it was shown that when the rotation angle is the golden ratio, that is, $\alpha=(\sqrt{5}-1) / 2$, the arithmetic average $(1 / n) \sum_{k=0}^{n-1} S_{k}(0)$ diverges logarithmically and this behavior is expected to persist for other quadratic irrationals. In the next two sections we will study the asymptotic behavior of $S_{n}$ from two different points of view. In Section 2, using an elementary argument which combines the Ostrowski representation of a positive integer and a version of the Denjoy-Koksma inequality we obtain general upper bounds (Theorem 2.1) for $\left\|S_{n}\right\|_{\infty}$. In Section 3, after having developed some general spectral theory we obtain several lower bounds both for $S_{n}$ and its Cesàro average in the $L^{2}$-norm (Theorems 3.9, 3.10 and 3.12). As a by-product we get a closed expression for the autocorrelation function of the function $\vartheta$ (Theorem 3.7) as well as estimates on some constrained sums (Proposition 3.6). The (upper and lower) bounds mentioned above turn out to depend intimately on the arithmetic properties of the rotation angle $\alpha$. These, in turn, are encoded by its continued fraction expansion, some properties of which will be recalled in the following subsection.

1.1. Continued fractions and return times. In this section we collect several known facts on continued fractions as well as some results on return times for ergodic translations to be used later on. To start with, let

$$
\alpha=\frac{1}{a_{1}+\frac{1}{a_{2}+\frac{1}{a_{3}+\cdots}}} \equiv\left[a_{1}, a_{2}, a_{3}, \ldots\right]
$$

be the continued fraction expansion of $\alpha$. One recursively constructs a sequence $p_{n} / q_{n}$ of rational approximants of $\alpha$ setting

$$
\frac{p_{0}}{q_{0}}=\frac{0}{1}, \quad \frac{p_{1}}{q_{1}}=\frac{1}{a_{1}}, \quad \frac{p_{n+1}}{q_{n+1}}=\frac{a_{n+1} p_{n}+p_{n-1}}{a_{n+1} q_{n}+q_{n-1}} .
$$

Then $p_{n} / q_{n}=\left[a_{1}, a_{2}, a_{3}, \ldots, a_{n}\right]$ and $p_{2 n} / q_{2 n}<\alpha<p_{2 n-1} / q_{2 n-1}$ for all $n>0$ (see [10, Chapter 1$])$. Given $u \in \mathbb{R}$ let $[u]$ and $\{u\}$ be its integer and fractional part, respectively. Let $G$ : $[0,1] \rightarrow[0,1]$ be the Gauss transformation given by $G(x)=\{1 / x\}$ for $x>0$ and $G(0)=0$. One readily sees that $\alpha=\left[a_{1}, a_{2}, \ldots, a_{n}+G^{n}(\alpha)\right]$, so that $a_{n+1}=\left[1 /\left(G^{n}(\alpha)\right)\right]$ or $G^{n}(\alpha)=$ $\left[a_{n+1}, a_{n+2}, \ldots\right]$ (we are making the conventions that $1 / 0=\infty$ and $1 / \infty=0$ ) and also

$$
\alpha=\frac{p_{n-1}+\left(G^{n}(\alpha)\right)^{-1} p_{n}}{q_{n-1}+\left(G^{n}(\alpha)\right)^{-1} q_{n}}
$$

which combined with Lagrange's formula $q_{n} p_{n-1}-q_{n-1} p_{n}=(-1)^{-n}$ yields

$$
\alpha-\frac{p_{n}}{q_{n}}=\frac{(-1)^{n}}{q_{n}\left(q_{n-1}+\left(G^{n}(\alpha)\right)^{-1} q_{n}\right)}=\frac{(-1)^{n}}{q_{n}\left(q_{n+1}+G^{n+1}(\alpha) q_{n}\right)} .
$$


4 Dispersion properties of ergodic translations

Now assume that $\alpha \in \mathbb{R} \backslash \mathbb{Q}$. In this case the iterative process $G^{n}(\alpha)$ is endless and for all $n \geq 1$ we have $a_{n+1}<\left(G^{n}(\alpha)\right)^{-1}<a_{n+1}+1$. One gets the well-known inequalitites

$$
\frac{1}{q_{n} \cdot\left(q_{n}+q_{n+1}\right)}<\left|\alpha-\frac{p_{n}}{q_{n}}\right|<\frac{1}{q_{n} \cdot q_{n+1}} .
$$

Moreover, since

$$
q_{n}+G^{n}(\alpha) q_{n-1}=\left(\prod_{k=0}^{n-1} G^{k}(\alpha)\right)^{-1}
$$

one has the bounds

$$
\frac{1}{2 \prod_{k=0}^{n-1} G^{k}(\alpha)}<q_{n}<\frac{1}{\prod_{k=0}^{n-1} G^{k}(\alpha)} .
$$

From (1.15) one deduces the following characterization for the denominators $q_{n}$ : let $|x|=$ $\min \{|x-p|: p \in \mathbb{Z}\}$ be the distance from the nearest integer, then

$$
q_{n}=\min \left\{r>q_{n-1}:\|r \alpha\|<\left\|q_{n-1} \alpha\right\|\right\}
$$

so that $\|r \alpha\| \geq\left\|q_{n-1} \alpha\right\|$ for all $r<q_{n}$ (see Figure 1.1).

If we denote by $d$ the Euclidean metric on $X$, then

$$
d\left(x, T^{r} x\right)=\min _{p \in \mathbb{Z}}|x+r \alpha-x-p|=\|r \alpha\|
$$

for all $x \in X$. Set

$$
f_{n}:=d\left(x, T^{q_{n}} x\right)=\left\|q_{n} \alpha\right\|=\left|q_{n} \alpha-p_{n}\right|=(-1)^{n}\left(q_{n} \alpha-p_{n}\right) .
$$

From the above one deduces the recursion formula

$$
f_{n-1}=a_{n+1} f_{n}+f_{n+1}
$$

and also

$$
f_{n}=\prod_{k=0}^{n} G^{k}(\alpha)
$$

Equation (1.22) shows that for all irrational $0<\alpha<1$ the sequence $f_{n}$ is strictly decreasing and by (1.15) satisfies the bounds

$$
\frac{1}{a_{n+1}+2}<q_{n} \cdot f_{n}<\frac{1}{a_{n+1}} .
$$




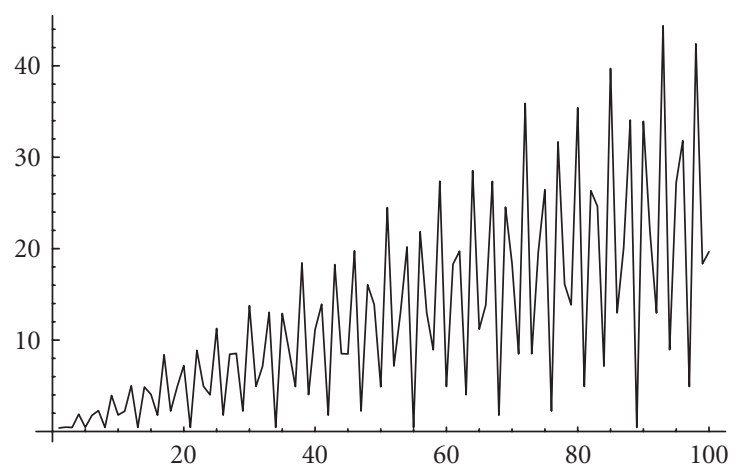

Figure 1.1. $r \cdot\|r \alpha\|$ versus $r$ for $\alpha=(\sqrt{5}-1) / 2$.

Finally, using (1.15) and (1.17) one can easily verify that the three conditions

$$
\begin{gathered}
r^{1+\eta} \cdot\|r \alpha\|>C_{1} \quad \forall r \geq 1, \\
q_{n+1}<C_{2} q_{n}^{1+\eta} \quad \forall n \geq 1, \\
\left(\prod_{k=0}^{n-1} G^{k}(\alpha)\right)^{\eta}<C_{3} G^{n}(\alpha) \quad \forall n \geq 1,
\end{gathered}
$$

where $C_{i}, i=1,2,3$, are positive constants and $\eta \geq 0$ are equivalent to one another, and the same equivalence is true for the complementary condition where the reverse inequalities are required to hold for infinite subsequences of values of $r$ and $n$.

Definition 1. The irrational number $\alpha$ is of type $\gamma$ if

$$
\gamma=\sup \left\{s: \lim _{r \rightarrow \infty} r^{s} \cdot\|r \alpha\|=0\right\}
$$

By (1.18) one has that if $q_{n} \leq r<q_{n+1}$, then $r^{s} \cdot\|r \alpha\| \geq q_{n}^{s} \cdot f_{n}$ for $s \geq 1$ so that the lower limit in (1.25) is reached along the subsequence $r=q_{n}$ for such values of $s$. On the other hand, by (1.23) we have $q_{n}^{1-\epsilon} \cdot f_{n}<1 / q_{n}^{\epsilon}$ and therefore $\gamma \geq 1$. Moreover, by (1.23) it follows that $\underline{\lim }_{r \rightarrow \infty} r \cdot\|r \alpha\|=0$ if and only if we can find an infinite subsequence $\left\{n_{k}\right\}$ such that $a_{n_{k}} \rightarrow \infty$. As an example consider the number $\alpha=e-2$. It is known [13] that in this case we have $a_{n}=1$ except for the infinite subsequence $n_{k}=3 k-1, k=1,2, \ldots$, with $a_{n_{k}}=2 k$. By $(1.23)$ we then have $1 / 2(k+2)<q_{3 k+1} \cdot f_{3 k+1}<1 / 2(k+1)$. On the other hand, (1.17) yields $q_{3 k+1} \geq \prod_{h=1}^{k} 2 h=2^{k} k$ ! and therefore $e-2$ is of type 1 .

The set of type 1 numbers has measure 1 and coincides with the set of Roth-type numbers. In particular it includes those with bounded partial quotients. One has (see [13, 4.5-4.6]) $\underline{\lim }_{r \rightarrow \infty} r \cdot\|r \alpha\| \leq 1 / \sqrt{m^{2}+1}$ for any $\alpha=\left[a_{1}, a_{2}, \ldots\right]$ unless $a_{i}<m$ for all $i$ large enough. At the opposite extreme, we say that $\alpha$ is well approximable by rational numbers if $\eta(\alpha)>0$ where $\eta(\alpha)$ is the supremum of all $\eta$ such that for all $k \geq 1$ we have $q_{k} \cdot\left\|q_{k} \alpha\right\|<$ $C q_{k}^{-\eta}$. In this case $\alpha$ is of type $1+\eta(\alpha)$. If, for example, $a_{k}=2^{2^{k}}$, then $\alpha$ is of type 2 . More 
generally, a number of type (at least) $\gamma \geq 1$ can be produced by constructing recursively its continued fraction expansion in such a way that at each step one has $a_{k} \geq\left[q_{k}^{\gamma-1}\right]$. This produces Diophantine as well as Liouville irrational numbers. A Diophantine number $\alpha$ of exponent $\eta \geq 0$, written $\alpha \in \mathrm{DC}(\eta)$, is such that $|\alpha-p / q| \geq C q^{-2-\eta}$ for all $p, q \in \mathbb{Z}$. Therefore it is of type (at most) $1+\eta$ (a Liouville number is "of type $\infty$ "). To be more precise, for $\gamma \geq 1$ the set of type $\gamma$ numbers coincides with $\cap_{\eta>\gamma} \mathrm{DC}(\eta+1) \backslash \cup_{\eta<\gamma} \mathrm{DC}(\eta+1)$.

We conclude with some standard and less standard results on return times. Let $\ell \in$ $(0,1 / 2]$ and set $t_{0}=0$ and $t_{i}=\inf \left\{k>t_{i-1}:\{k \alpha\}<\ell\right\}$, for $i>0$, that is, $\left\{t_{i}\right\}_{i \geq 0}$ is the sequence of entrance times in the half-open interval $[0, \ell)$ of the forward orbit of 0 under $T$. The sequence of return times $r_{i}=t_{i+1}-t_{i}$ determines a set of gaps, that is, the set of all possible values of $r_{i}, i \geq 0$. The frequency of a gap is defined as its frequency in the sequence $\left\{r_{i}\right\}_{i \geq 0}$. A classical result is the three-gap theorem (see [14, 6], [2, Section 4]). Let us first observe that there exists a unique expression of $\ell$ as

$$
\ell=c f_{k}+f_{k+1}+g \text { for some } k \geq 1,0<g \leq f_{k}, 1 \leq c \leq a_{k+1}
$$

where, by (1.21), the integer $k$ is uniquely determined by the inequalities

$$
f_{k}+f_{k+1}<\ell \leq f_{k-1}+f_{k}
$$

Indeed, the Euclidean division of $\ell$ by $f_{k}$ gives a quotient $c_{k} \leq a_{k+1}+1$ and a remainder $\ell_{k} \leq f_{k+1}$. Thus $\ell=c_{k} f_{k}+\ell_{k}=c f_{k}+f_{k+1}+g$ with $g=f_{k}-f_{k+1}+\ell_{k} \leq f_{k}$ and $c=c_{k}-1$.

The three-gap theorem then says that the gaps assume at most three values, one being the sum of the other two: $q_{k}$ (with frequency $(c-1) f_{k}+f_{k+1}+g$ ), $q_{k+1}-c q_{k}$ (with frequency $g$ ), and $q_{k+1}-(c-1) q_{k}$ (with frequency $f_{k}-g$ ).

By uniform distribution this result still holds when considering the gaps between the successive passages into any half-open interval $I$ with $|I|=\ell$.

Now let

$$
k_{n}:=\min \left\{k:\left\|q_{k} \alpha\right\|<\frac{1}{2 n}\right\}=\max \left\{k:\left\|q_{k-1} \alpha\right\| \geq \frac{1}{2 n}\right\} .
$$

From (1.12) one obtains the uniform lower bound $q_{k}>2^{k-1}$ so that (1.15) yields $\left\|q_{k-1} \alpha\right\|<$ $q_{k}^{-1}<2^{-k+1}$. Therefore the sequence $k_{n}$ cannot grow faster than $\log n$. Moreover, by $(1.18)$ we have

$$
\|r \alpha\| \geq\left\|q_{k_{n}-1} \alpha\right\| \geq \frac{1}{2 n} \quad \forall r<q_{k_{n}}
$$

Therefore, by (1.19), $q_{k_{n}}$ can be interpreted as the first time the orbit returns into a neighborhood of radius $1 / n$ of the initial point. Note that

$$
2 f_{\ell+1}<\frac{1}{n} \leq 2 f_{\ell} \Rightarrow k_{n}=\ell+1
$$


Lemma 1.1. For $q_{\ell} \leq n<q_{\ell+1}$ the function $k_{n}$ (and thus the return time $q_{k_{n}}$ ) may take at most three values: $k_{n} \in\{\ell, \ell+1, \ell+2\}$.

Proof. By the (inmost) inequality (1.15) we have that if $n \geq q_{\ell}$, then $f_{\ell-1}>1 /\left(2 q_{\ell}\right) \geq$ $1 /(2 n)$, thus $k_{n} \geq \ell$. Moreover, using (1.23) we see that if $n<q_{\ell+1}$, then $f_{\ell+1}<1 /\left(a_{\ell+2} q_{\ell+1}\right)<$ $1 /\left(a_{\ell+2} n\right)$. Therefore if $a_{\ell+2}>1$, then $k_{n} \leq \ell+1$. If instead $a_{\ell+2}=1$, then $f_{\ell+2}<1 / q_{\ell+3}<$ $1 /\left(2 q_{\ell+1}\right)<1 /(2 n)$.

Remark 1.2. Due to (1.30) we see that if $1 /\left(2 f_{\ell}\right) \leq q_{\ell}$ and $q_{\ell+1} \leq 1 /\left(2 f_{\ell+1}\right)$, then for $n$ ranging as $q_{\ell} \leq n<q_{\ell+1}$ the function $k_{n}$ takes on but the intermediate value $\ell+1$. In turn, by (1.23), for the first inequality to be satisfied it is necessary that $a_{\ell+1}=1$, while for the second it is sufficient that $a_{\ell+2}>1$.

Remark 1.3. We point out that the three times dealt with in Lemma 1.1 are the three possible values of the first time the orbit comes as close to the initial point as (at least) $1 / n$, when $n$ ranges in the interval $\left[q_{\ell}, q_{\ell+1}\right)$. A connection with the three-gap theorem is as follows: if $k$ is chosen so as to satisfy

$$
q_{\ell} \leq n=\left(c f_{k}+f_{k+1}+g\right)^{-1}<q_{\ell+1}
$$

with $1 \leq c \leq a_{k+2}$ and $0<g \leq f_{k}$, then $\{k\} \cap\{\ell, \ell+1, \ell+2\} \neq \varnothing$.

Consider, for example, the golden mean $\alpha=(\sqrt{5}-1) / 2=[1,1,1, \ldots]$. In this case $q_{k-1}=\left(\alpha^{-k}-(-\alpha)^{k}\right) / \sqrt{5}$ are the Fibonacci numbers whereas $f_{k-1}=\alpha^{k}$ by (1.22). Thus

$$
\frac{1}{f_{\ell-2}}<\frac{1}{2 f_{\ell-1}}<q_{\ell}<\frac{1}{f_{\ell-1}}<\frac{1}{2 f_{\ell}}<q_{\ell+1}<\frac{1}{f_{\ell}}
$$

and $k_{n}=\ell$ if $q_{\ell} \leq n<1 /\left(2 f_{\ell}\right)$ whereas $k_{n}=\ell+1$ if $1 /\left(2 f_{\ell}\right) \leq n<q_{\ell+1}$. From this one easily sees that $k_{n} \asymp \log n$ (we write $a_{n} \asymp b_{n}$ whenever there are constants $c_{1}$ and $c_{2}$ such that $c_{1} b_{n} \leq a_{n} \leq c_{2} b_{n}$ for all $\left.n\right)$. On the other hand, the condition (1.31) becomes $q_{\ell} \leq$ $n=\left(f_{k-1}+g\right)^{-1}<q_{\ell+1}$. For these values of $n$ the gaps between successive returns into an interval of size $1 / n$ are $q_{k-1}, q_{k}$, and $q_{k+1}$. By (1.27) $k$ is determined by the inequalities $f_{k-2}^{-1} \leq n<f_{k-1}^{-1}$. Moreover $f_{k-2}^{-1}<q_{k}<f_{k-1}^{-1}$. Therefore the above inequalities can be satisfied choosing either $k=\ell$ or $k=\ell+1$.

We finally characterize the asymptotic behavior of the first return time $q_{k_{n}}$ in terms of the type of the rotation angle $\alpha$.

LEMMA 1.4. If $\alpha$ is of type $\gamma \geq 1$ then

$$
\lim _{n \rightarrow \infty} \frac{\log q_{k_{n}}}{\log n}=\frac{1}{\gamma} .
$$

Proof. Under the hypothesis of the lemma we have $r^{\gamma+\epsilon} \cdot\|r \alpha\|>A_{\epsilon}$ for all $r \geq 1$ and some positive constant $A_{\epsilon}$. We thus get

$$
q_{k_{n}}^{\gamma+\epsilon} \geq \frac{A_{\epsilon}}{\left\|q_{k_{n}} \alpha\right\|}>2 A_{\epsilon} n
$$


so that $\underline{\lim }_{n \rightarrow \infty} \log q_{k_{n}} / \log n>1 /(\gamma+\epsilon)$. By the arbitrariness of $\epsilon$ we thus have

$$
\lim _{n \rightarrow \infty} \frac{\log q_{k_{n}}}{\log n} \geq \frac{1}{\gamma}
$$

In the other direction, we have $\underline{\lim }_{r \rightarrow \infty} r^{\gamma-\epsilon} \cdot\|r \alpha\|=0$ and therefore we can find an infinite subsequence $\left\{q_{j}\right\}$ so that $q_{j}^{\overline{\gamma-\epsilon}} \cdot\left\|q_{j} \alpha\right\|<1$. Choose a sequence $\left\{n_{j}\right\}$ such that $2 n_{j}<$ $q_{j}^{\gamma-\epsilon} \leq 4 n_{j}$ for all $j$. It then follows that $\left\|q_{j} \alpha\right\|<1 / 2 n_{j}$ and therefore $q_{k_{n_{j}}} \leq q_{j} \leq\left(4 n_{j}\right)^{1 /(\gamma-\epsilon)}$. Reasoning as above completes the proof.

\section{The asymptotic growth of $S_{n}$}

In this section we study the asymptotic growth of the sums $S_{n}$ defined in (1.4) in $L^{\infty}$ norm. The asymptotic behavior of $S_{n}$ and its average $1 / n \sum_{k=0}^{n-1} S_{k}(x)$ in $L^{2}$-norm will be dealt with in Section 3 using spectral techniques.

To warm up let us consider $\alpha$ rational, say $\alpha=p / q$. We can reason as follows: the unit circle gets partitioned by the equidistant points $0,1 / q, \ldots, q-1 / q$ and for any choice of the initial point $x$ the piece of orbit $\{x+k \alpha\}_{0 \leq k<q}$ visits each subset $[i / q,(i+1) / q), i=$ $0, \ldots, q-1$, exactly once (the order of the visits being determined by $p$ ). A short reflection shows that the discrepancy between the number of times this orbit lies in $A_{+}$and in $A_{-}$ is bounded by 2. More precisely, if $q$ is even, then this discrepancy is zero, whereas for $q$ odd there is $i$ such that $i / q<1 / 2<(i+1) / q$ and the point may fall either in $[i / q, 1 / 2)$ or in $[1 / 2,(i+1) / q)$. This yields, for all $k \geq 1$,

$$
\left\|S_{k \cdot q}\right\|_{\infty}= \begin{cases}2 k & \text { if } \mathrm{q} \text { is odd } \\ 0 & \text { if } \mathrm{q} \text { is even. }\end{cases}
$$

Thus, for rational $\alpha$ one has a nondiffusive behavior.

For $\alpha$ irrational we may consider its continued fraction expansion (1.11) along with the sequence of its rational approximants $p_{\ell} / q_{\ell}=\left[a_{1}, a_{2}, \ldots, a_{\ell}\right]$. Inequality $(1.15)$ yields

$$
\left|k \alpha-k \frac{p_{\ell}}{q_{\ell}}\right|<\frac{k}{q_{\ell}^{2} \cdot a_{\ell+1}}<\frac{1}{q_{\ell} \cdot a_{\ell+1}}, \quad 1 \leq k \leq q_{\ell} .
$$

Therefore for each $\ell \in \mathbb{N}$ the unit circle gets partitioned into the subsets $\left[i / q_{\ell},(i+1) / q_{\ell}\right)$, $i=0, \ldots, q_{\ell}-1$, and the piece of orbit $\{x+k \alpha\}_{0 \leq k<q_{\ell}}$ is fairly well distributed among them. More specifically, using (2.2) one realizes that the discrepancy between the number of times the orbit lies in $A_{+}$and in $A_{-}$is bounded above by 2 . We then get

$$
\left\|S_{k \cdot q_{\ell}}\right\|_{\infty} \leq 2 k, \quad k \geq 1
$$

In particular, the sum $S_{n}$ is uniformly bounded along the subsequence $\left\{q_{\ell}\right\}$. But the latter has (at least) exponentially large gaps and the absence of mixing for the sequence $\left\{\vartheta \circ T^{k}\right\}$ 
may allow for a growth behavior of $\max _{q_{\ell} \leq n<q_{\ell+1}}\left|S_{n}\right|$ (for sums of i.i.d. random variables it is well known that if $\max _{1 \leq n \leq N}\left|S_{n}\right|$ is large, then with high probability $\left|S_{N}\right|$ is large as well).

THeOREM 2.1. If $\alpha$ has bounded partial quotients, then for $q_{\ell} \leq n<q_{\ell+1}$,

$$
\left\|S_{n}\right\|_{\infty}=O(\log n)
$$

If $\alpha$ is of type $\gamma \geq 1$, then for all $\epsilon>0$,

$$
\left\|S_{n}\right\|_{\infty}=O\left(n^{1-(1 /(\gamma+\epsilon))} \log n\right)
$$

If moreover $\alpha \in D C(\eta)$ with $\eta \geq 0$, then

$$
\left\|S_{n}\right\|_{\infty}=O\left(n^{1-(1 /(\eta+1))} \log n\right) .
$$

Proof. For $q_{\ell} \leq n<q_{\ell+1}$ we may use the Ostrowski representation for $n$ (see [13, Chapter II.4]):

$$
n=\sum_{k=0}^{\ell} c_{k} q_{k} \quad \text { with } 0 \leq c_{k} \leq a_{k+1}, c_{k-1}=0 \quad \text { if } c_{k}=a_{k+1} .
$$

Using this and (2.3) we get

$$
\left\|S_{n}\right\|_{\infty} \leq \sum_{k=0}^{\ell}\left\|S_{c_{k} q_{k}}\right\|_{\infty} \leq 2 \sum_{k=0}^{\ell} c_{k}, \quad q_{\ell} \leq n<q_{\ell+1} .
$$

If the partial quotients of $\alpha$ are uniformly bounded (so that it is of type 1) this gives

$$
\left\|S_{n}\right\|_{\infty}=O(\log n)
$$

since $\ell=O(\log n)$. If $\alpha$ is of type $\gamma \geq 1$, then for each $\epsilon>0$ we have $r^{\gamma+\epsilon} \cdot\|r \alpha\|>A_{\epsilon}$ for all $r \geq 1$ and some positive constant $A_{\epsilon}$. Using (1.24) we see that we can find a positive constant $B_{\epsilon}$ so that $q_{\ell+1}<B_{\epsilon} q_{\ell}^{\gamma+\epsilon}$. Therefore $q_{\ell} \leq n<B_{\epsilon} q_{\ell}^{\gamma+\epsilon}$. From this and (2.7) we obtain $c_{\ell} \leq n / q_{\ell}<B_{\epsilon}^{1 /(\gamma+\epsilon)} n^{1-(1 /(\gamma+\epsilon))}$ and one sees inductively that the same inequality holds true for $c_{k}$ with $k=0, \ldots, \ell$. Indeed, write $n=c_{\ell} q_{\ell}+n^{\prime}$ with $n^{\prime}=\sum_{k=0}^{\ell-1} c_{k} q_{k}$. Reasoning as above we get $c_{\ell-1} \leq n^{\prime} / q_{\ell-1}<B_{\epsilon}^{1 /(\gamma+\epsilon)}\left(n^{\prime}\right)^{1-1 /(\gamma+\epsilon)}<B_{\epsilon}^{1 /(\gamma+\epsilon)} n^{1-1 /(\gamma+\epsilon)}$, and so on. This yields the estimate $(2.5)$. The last statement follows immediately by noting that being Diophantine of exponent $\eta \geq 0$ is equivalent to the statement $q_{\ell+1}=O\left(q_{\ell}^{\eta+1}\right)$.

Remark 2.2. Theorem 2.1 can be extended in various directions. For instance, one may consider irrational numbers $\alpha$ such that $q_{\ell+1}<e^{\lambda q_{\ell}^{1 / \delta}}$ for some positive constants $\lambda$ and $\delta$ 


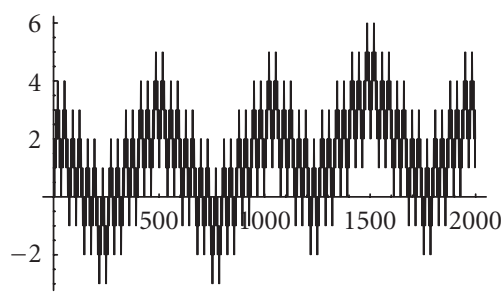

(a)

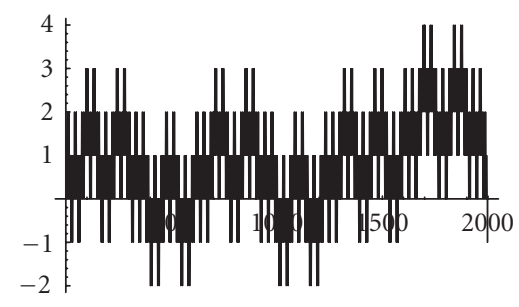

(b)

Figure 2.1. $S_{n}(0)$ versus $n$ for (A) $\alpha=e-2=[1,2,1,1,4,1,1,6, \ldots]$ and (B) $\alpha=(\sqrt{5}-1) / 2=$ $[1,1,1, \ldots]$.

(such numbers may be "of type $\infty$ "). Reasoning as in the proof of the above theorem we get in this case the estimate

$$
\left\|S_{n}\right\|_{\infty}=O\left(\frac{n}{\log ^{\delta} n}\right) .
$$

Furthermore, the estimate (2.5) can be improved when some additional knowledge on the partial quotients is available. Consider again the number $\alpha=e-2$ (which is of type 1). In this case, using (2.8) along with the explicit expression for the partial quotients mentioned in the previous section, we get $\left\|S_{n}\right\|_{\infty} \leq 2 \sum_{i=1}^{3 k+1} a_{i}=2\left(k^{2}+3 k+1\right)$ for all $q_{3 k} \leq n<q_{3 k+1}$. On the other hand, since $q_{3 k+1} \geq 2^{k} k$ ! and $q_{3 k} \geq 2 q_{3 k-2} \geq 2^{k}(k-1)$ !, the condition $q_{3 k} \leq n<q_{3 k+1}$ implies $2^{k}(k-1) ! \leq n<2^{k} k$ !. A simple computation then yields $k=O(\log n / \log \log n)$. Therefore, for $n$ as above we find

$$
\left\|S_{n}\right\|_{\infty}=O\left(\frac{\log ^{2} n}{\log ^{2} \log n}\right) .
$$

Some insight to understand the pictures in Figure 2.1 may be achieved by noting that by the three-gap theorem in the symbolic sequence $\pi(x)$ the blocks of consecutive 1's (or -1 's) have at most three lengths, for all $x \in X$. As an example consider the golden ratio $\alpha=(\sqrt{5}-1) / 2$. Here we find

$$
f_{1}=1-\alpha \simeq 0.38, \quad f_{2}=2 \alpha-1 \simeq 0.24, \quad f_{3}=2-3 \alpha \simeq 0.14
$$

Therefore $\left|A_{+}\right|=1 / 2=f_{1}+g$ with $g=\alpha-1 / 2 \simeq 0.12$. The gaps, and thus the lengths of the blocks mentioned above, are then $q_{1}=1, q_{2}=2$, and $q_{3}=3$ with frequencies $g$, $g+f_{3}$, and $f_{2}-g$, respectively (these are the lengths of the subintervals of $A_{+}$on which the return time is constant and one easily checks that they sum up to $1 / 2$ ).

We conclude this section with two more remarks.

Remark 2.3. The proof of the upper estimates obtained above relies on the Ostrowski representation of a positive integer along with the basic inequality (2.3). On the other 
hand, the latter is a particular case of the Denjoy-Koksma inequality (see [11]): let $f$ : $X \rightarrow \mathbb{R}$ have bounded variation and satisfy $\int_{X} f(x) \mu(d x)=0$. Then for $\alpha \in \mathbb{R} \backslash \mathbb{Q}$,

$$
\left|\sum_{k=0}^{q_{n}-1} f(x+k \alpha)\right| \leq 2 \operatorname{Var}(f) .
$$

Thus the results stated above can be straightforwardly extended to any function $f$ with bounded variation. However, as explained in the introduction, here we are mainly interested in studying diffusion properties of the random walk on $\mathbb{Z}$ which arises using the particular function $\vartheta$.

Remark 2.4. The behavior of the sequence $S_{n}(x)$ determines the analytic structure of the generating function

$$
F(\alpha, x, z):=\sum_{n \geq 1} \vartheta(x+n \alpha) z^{n}
$$

In particular, the asymptotic growth of $S_{n}(x)$ determines the strength of the singularity of $F(\alpha, x, z)$ at $z=1$. One moreover easily realizes that for each irrational $\alpha$ and all $x \in X$ the function $F(\alpha, x, z)$ has its circle of convergence (which in this case is the unit circle) as a natural boundary, a property shared (with probability one) by Taylor series whose coefficients are symmetric random variables [9]. Set indeed $z=r e^{2 \pi i \theta}$ with $0 \leq r \leq 1$ and take $\theta=q \alpha(\bmod 1)$ with $q \neq 0$. By unique ergodicity we have

$$
\lim _{n \rightarrow \infty} \frac{1}{n} \sum_{k=0}^{n-1} \vartheta(x+k \alpha) e^{2 \pi i k q \alpha}=e^{-2 \pi i q x} \int_{0}^{1} \vartheta(y) e^{2 \pi i q y} d y= \begin{cases}\frac{2}{i q \pi} e^{-2 \pi i q x}, & \text { q odd } \\ 0, & \text { q even. }\end{cases}
$$

Therefore, by Abel's second theorem [12, Theorem 5.2] we have

$$
\lim _{r \rightarrow 1_{-}}(1-r) F\left(\alpha, x, r e^{2 \pi i q \alpha}\right)= \begin{cases}\frac{2}{i q \pi} e^{-2 \pi i q x}, & \text { q odd }, \\ 0, & \text { q even. }\end{cases}
$$

\section{The spectral approach}

We will now investigate the average behavior of $\left|S_{n}\right|$ by means of spectral techniques. Given $f: X \rightarrow \mathbb{R}$ denote by $\mu(f)$ the expectation $\int_{X} f(x) \mu(d x)$. As already noted $\mu\left(S_{n}\right)=0$. Diffusion properties of the walk are described by the dispersion $D S_{n}$ given by

$$
D S_{n}=\mu\left(S_{n}^{2}\right)-\mu\left(S_{n}\right)^{2}=\mu\left(S_{n}^{2}\right) \equiv\left\|S_{n}\right\|_{2}^{2} .
$$

We preliminary note that since

$$
D S_{n}=\int_{X} S_{n}^{2}(x) \mu(d x) \leq\left\|S_{n}\right\|_{\infty}^{2},
$$

an immediate consequence of Theorem 2.1 is the following estimate. 
Corollary 3.1. If $\alpha$ has bounded partial quotients, then

$$
D S_{n}=O\left(\log ^{2} n\right)
$$

If $\alpha$ is of type $\gamma \geq 1$, then for each $\epsilon>0$,

$$
D S_{n}=O\left(n^{2(1-1 /(\gamma+\epsilon))} \log ^{2} n\right) .
$$

If moreover $\alpha \in D C(\eta)$ with $\eta \geq 0$, then

$$
D S_{n}=O\left(n^{2(1-1 /(\eta+1))} \log ^{2} n\right) .
$$

In order to obtain lower bounds we will first develop some general spectral theory which will be then applied to our setting.

3.1. Some general spectral theory. Although what follows could be easily formulated for any $f: X \rightarrow \mathbb{R}$, for the sake of simplicity we will only consider the special case $f=\vartheta$. Other material on this subject can be found in $[4,8]$. Let $T: X \rightarrow X$ be homeomorphism of the circle and $\mu$ an ergodic $T$-invariant probability measure on $X$. We write

$$
\begin{aligned}
D S_{n} & =\int_{X} \sum_{l, j=0}^{n-1} \vartheta\left(T^{l}(x)\right) \vartheta\left(T^{j}(x)\right) \mu(d x) \\
& =\int_{X} \sum_{l, j=0}^{n-1} \vartheta(x) \vartheta\left(T^{j-l}(x)\right) \mu(d x)=\sum_{k=-n+1}^{n-1}(n-|k|) b_{k}(\vartheta),
\end{aligned}
$$

where we have introduced the autocorrelation function $b_{k}(\vartheta)$ defined by $b_{0}(\vartheta)=1$ and

$$
b_{k}(\vartheta)=\int_{X} \vartheta(x) \vartheta\left(T^{k}(x)\right) \mu(d x) \equiv \mu\left(\vartheta \cdot \vartheta \circ T^{k}\right)
$$

$T$ being invertible, the function $b_{k}(\vartheta)$ satisfies $b_{k}(\vartheta)=b_{-k}(\vartheta)$. An interesting quantity is the spectral type of $\vartheta$, that is, the probability measure $\sigma_{\vartheta}$ on $(0,1]$ such that

$$
b_{k}(\vartheta)=\int_{0}^{1} e^{2 \pi i k \lambda} \sigma_{\vartheta}(d \lambda)
$$

By means of this measure we can express $D S_{n}$ in the following way.

Proposition 3.2.

$$
D S_{n}=\int_{0}^{1} \Phi_{n}(x) \sigma_{\vartheta}(d x)
$$

where

$$
\Phi_{n}(x):=\frac{\sin ^{2} n \pi x}{\sin ^{2} \pi x}
$$


Proof. The assertion follows upon combining the identity

$$
\sum_{k=-n+1}^{n-1}(n-|k|) e^{i k t}=\frac{\sin ^{2}(n t / 2)}{\sin ^{2}(t / 2)}
$$

with (3.6) and (3.8).

Notice that the function $\Phi_{n}(x)$ is 1-periodic and satisfies

$$
\int_{0}^{1} \Phi_{n}(x) d x=n
$$

We now show that the Cesàro means

$$
\left\langle D S_{n}\right\rangle:=\frac{1}{n} \sum_{k=0}^{n-1} D S_{k}
$$

always have a limit (finite or infinite). More specifically we have the following.

Proposition 3.3.

$$
\lim _{n \rightarrow \infty}\left\langle D S_{n}\right\rangle=\int_{0}^{1} \frac{\sigma_{9}(d x)}{2 \sin ^{2} \pi x}
$$

Proof. A straightforward computation yields for the function defined in (3.10)

$$
\left\langle\Phi_{n}(x)\right\rangle:=\frac{1}{n} \sum_{k=0}^{n-1} \Phi_{k}(x)=\frac{1}{\sin ^{2} \pi x}\left[\frac{1}{2}-\frac{1}{4 n}\left(1+\frac{\sin (2 n-1) \pi x}{\sin \pi x}\right)\right]
$$

which converges to $1 / 2 \sin ^{2} \pi x$ when $n \rightarrow \infty$. Upon observing that for each fixed $x \in(0,1)$ the expression between inner brackets is periodic in $n$ (with period $1 / x$ ) one readily shows using Lebesgue's monotone convergence theorem that $\underline{\lim }\left\langle\Phi_{n}(x)\right\rangle=\overline{\lim }\left\langle\Phi_{n}(x)\right\rangle$ and both are equal to the integral in (3.14).

We point out that (3.9), (3.10), and (3.14) are general formulas for the dispersion, the dependence on the transformation $(T, \mu)$ being entirely contained in the spectral measure

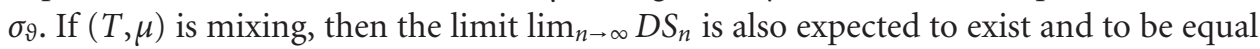
to $(3.14)$.

Example 3.4. In the case of an endomorphism $T: X \rightarrow X$ the autocorrelation sequence $b_{k}(\vartheta)=\mu\left(\vartheta \cdot \vartheta \circ T^{k}\right)$ is defined only for $k \geq 0$. On the other hand, for negative $k$ one may put $b_{k}=\bar{b}_{-k}$ so that (3.6) and (3.8) remain valid (see [4]). For example, for the two-toone map of the circle given by $T(x)=2 \cdot x(\bmod 1)$ we find $\vartheta\left(T^{k}(x)\right)=\sum_{r \text { odd }}(2 / \operatorname{ir} \pi) \chi_{2^{k} r}(x)$ with $\chi_{r}(x):=e^{2 \pi i r x}$ and therefore $b_{k}(\vartheta)=\delta_{k, 0}$ or, which is the same, the spectral measure $\sigma_{\vartheta}$ is but the Lebesgue measure. Therefore from (3.9) and (3.12) we get $D S_{n}=n$ for all $n \geq 1$. We point out that in this case we have $S_{n}=\sum_{k=1}^{n} \epsilon_{k}$ where $\epsilon_{k}$ is the Rademacher 
sequence defined on the standard Lebesgue probability space. Whence one has the estimate (see $[9$, page 8$])$ :

$$
\mu\left(S_{n}^{2}>a n\right)>\frac{(1-a)^{2}}{3} \text { for } 0<a<1
$$

The purely linear behavior $D S_{n}=n$ persists for the map $T(x)=m \cdot x(\bmod 1)$ provided $m$ is an even (positive) integer. For $m$ odd we find $b_{k}(\vartheta)=m^{-k}$ and a short calculation gives the spectral density

$$
\begin{gathered}
\rho(\lambda):=\frac{d \sigma_{\vartheta}(\lambda)}{d \lambda}=\frac{m^{2}-1}{m^{2}-2 m \cos 2 \pi \lambda+1}, \\
D S_{n}=\left(\frac{m+1}{m-1}\right) n-\frac{2 m\left(1-m^{-n}\right)}{(m-1)^{2}}=\rho(0) n+o(n),
\end{gathered}
$$

to be compared to [8, Theorem 6].

We now derive general bounds which relate the asymptotic behavior of $D S_{n}$ and $\left\langle D S_{n}\right\rangle$ to the behavior of the spectral measure $\sigma_{\vartheta}$ near the endpoints 0 and 1 .

In order to estimate $\Phi_{n}(x)$ and $\left\langle\Phi_{n}(x)\right\rangle$ let us recall from basic calculus that

$$
\frac{2}{\pi} \leq \frac{\sin \pi x}{\pi x} \leq 1 \quad \forall 0 \leq x \leq \frac{1}{2}
$$

where the upper bound holds for all $x \in \mathbb{R}_{+}$. This yields the following bounds:

$$
\begin{array}{cc}
\frac{4}{\pi^{2}} n^{2} \leq \Phi_{n}(x) \leq \frac{\pi^{2}}{4} n^{2} & \text { for } 0 \leq x \leq \frac{1}{2 n}, \\
\Phi_{n}(x) \leq \frac{1}{\sin ^{2} \pi x} \leq \frac{1}{4 x^{2}} & \text { for } \frac{1}{2 n} \leq x \leq \frac{1}{2} .
\end{array}
$$

Moreover, by (3.15) we can easily find two positive constants $C_{1}$ and $C_{2}$ so that

$$
C_{1} n^{2} \leq\left\langle\Phi_{n}(x)\right\rangle \leq C_{2} n^{2} \quad \text { for } 0 \leq x \leq \frac{1}{2 n} .
$$

Using again (3.18), we find

$$
\left|\frac{\sin (2 n-1) \pi x}{\sin \pi x}\right| \leq \frac{1}{\sin \pi x} \leq n \quad \text { for } \frac{1}{2 n} \leq x \leq \frac{1}{2},
$$

whence

$$
\begin{aligned}
& \left|\frac{1}{2}-\frac{1}{4 n}\left(1+\frac{\sin (2 n-1) \pi x}{\sin \pi x}\right)\right| \geq \frac{1}{4}\left(1-\frac{1}{n}\right) \geq \frac{1}{8} \quad \forall n>1, \\
& \left|\frac{1}{2}-\frac{1}{4 n}\left(1+\frac{\sin (2 n-1) \pi x}{\sin \pi x}\right)\right| \leq \frac{3}{4}\left(1-\frac{1}{3 n}\right) \leq \frac{3}{4} \quad \forall n \geq 1 .
\end{aligned}
$$


Therefore we have the bounds

$$
\frac{1}{8 \sin ^{2} \pi x} \leq\left\langle\Phi_{n}(x)\right\rangle \leq \frac{3}{4 \sin ^{2} \pi x} \quad \text { for } \frac{1}{2 n} \leq x \leq \frac{1}{2} .
$$

We point out that the behavior of the functions $\Phi_{n}(x)$ and $\left\langle\Phi_{n}(x)\right\rangle$ is similar in the region $0 \leq x \leq 1 / 2 n$ where they are both of order $n^{2}$. Instead for $1 / 2 n \leq x \leq 1 / 2$ their difference becomes evident in that unlike $\Phi_{n}(x),\left\langle\Phi_{n}(x)\right\rangle$ never vanishes (compare (3.20) and (3.24)).

Setting $A_{n}=[0,1 / 2 n] \cup[1-1 / 2 n, 1]$ and $B_{n}=[1 / 2 n, 1-1 / 2 n]$, we write

$$
\begin{gathered}
D S_{n}=\int_{A_{n}} \Phi_{n}(x) \sigma_{\vartheta}(d x)+\int_{B_{n}} \Phi_{n}(x) \sigma_{\vartheta}(d x)=: I_{n}+J_{n} \\
\left\langle D S_{n}\right\rangle=\int_{A_{n}}\left\langle\Phi_{n}(x)\right\rangle \sigma_{\vartheta}(d x)+\int_{B_{n}}\left\langle\Phi_{n}(x)\right\rangle \sigma_{\vartheta}(d x)=: I_{n}^{\prime}+J_{n}^{\prime} .
\end{gathered}
$$

According to the above discussion, we expect the integrals $I_{n}$ and $I_{n}^{\prime}$ to behave similarly, the same not being true for $J_{n}$ and $J_{n}^{\prime}$.

To see this, let us define the spectral coefficients $\sigma_{k}(k \geq 1)$ as

$$
\sigma_{k}:=\sigma_{\vartheta}\left(\left[\frac{1}{2(k+1)}, \frac{1}{2 k}\right] \cup\left[1-\frac{1}{2 k}, 1-\frac{1}{2(k+1)}\right]\right) .
$$

Then (3.19) gives

$$
I_{n} \asymp I_{n}^{\prime} \asymp n^{2} \sum_{k \geq n} \sigma_{k} .
$$

On the other hand, (3.20) yields the upper bound

$$
J_{n} \leq \int_{B_{n}} \frac{\sigma_{\vartheta}(d x)}{4 x^{2}} \leq \sum_{k=1}^{n}(k+1)^{2} \sigma_{k}=O\left(\sum_{k=1}^{n} k^{2} \sigma_{k}\right),
$$

whereas (3.24) and (3.18) give

$$
J_{n}^{\prime} \asymp \int_{B_{n}} \frac{\sigma_{\vartheta}(d x)}{x^{2}} \asymp \sum_{k=1}^{n} k^{2} \sigma_{k} .
$$

We summarize the above as follows.

Proposition 3.5.

$$
D S_{n}=O\left(n^{2} \sum_{k \geq n} \sigma_{k}+\sum_{k=1}^{n} k^{2} \sigma_{k}\right)
$$

and, for $n>1$,

$$
\left\langle D S_{n}\right\rangle \asymp n^{2} \sum_{k \geq n} \sigma_{k}+\sum_{k=1}^{n} k^{2} \sigma_{k}
$$


By a straightforward extension of a number-theoretic lemma proved in [8, page 662] if $\left\{\sigma_{k}\right\}_{k \geq 1}$ is a sequence of nonnegative real numbers and $t \in[0,2)$, then for $n \rightarrow \infty$

$$
\sum_{k \geq n} \sigma_{k}=O\left(n^{-t} L(n)\right) \Longrightarrow \sum_{k=1}^{n} k^{2} \sigma_{k}=O\left(n^{2-t} L(n)\right),
$$

where $L(n)$ is a slowly varying function, that is, $L(c n) \sim L(n)$ as $n \rightarrow \infty$. When $t>0$ the reverse implication is also valid. This entails that whenever the above conditions are satisfied the two terms in the right-hand side of (3.31) (or (3.32)) give the same upper bound for $D S_{n}$ (and thus for $\left\langle D S_{n}\right\rangle$ ). Conversely, putting together (3.19) and (3.25) we obtain

$$
\sum_{k \geq n} \sigma_{k} \equiv \sigma_{\vartheta}\left(\left[0, \frac{1}{2 n}\right] \cup\left[1-\frac{1}{2 n}, 1\right]\right) \leq \frac{\pi^{2}}{4} \frac{I_{n}}{n^{2}} \leq \frac{\pi^{2}}{4} \frac{D S_{n}}{n^{2}} .
$$

Of course a similar estimate can be obtained for $\left\langle D S_{n}\right\rangle$ using (3.21).

3.2. The case of rotations. For the map $T x=x+\alpha(\bmod 1)$ it is well known that the unitary operator $U_{\alpha}: L^{2}(X, \mu) \rightarrow L^{2}(X, \mu)$ acting as $U_{\alpha} f=f \circ T$ has a pure point spectrum [4], with eigenvalues $\lambda_{r}$ and eigenfunctions $\chi_{r}$, given by

$$
\lambda_{r}=e^{2 \pi i r \alpha}, \quad \chi_{r}(x)=e^{2 \pi i r x}, \quad r \in \mathbb{Z} .
$$

Let $(f, g)=\int_{S^{1}} f(x) \overline{g(x)} \mu(d x)$ be the inner product of $f, g \in L^{2}\left(S^{1}, \mu\right)$. An easy computation yields

$$
\left(\vartheta, \chi_{r}\right)=\frac{1-2 e^{-\pi i r}+e^{-2 \pi i r}}{2 \pi i r}= \begin{cases}\frac{2}{i r \pi}, & \text { r odd } \\ 0, & \text { r even }\end{cases}
$$

Therefore we have the expansions

$$
\vartheta(x)=\sum_{r \text { odd }}\left(\frac{2}{i r \pi}\right) \chi_{r}(x), \quad \vartheta\left(R_{\alpha}^{k}(x)\right)=\sum_{r \text { odd }}\left(\frac{2 e^{2 \pi i k r \alpha}}{i r \pi}\right) \chi_{r}(x),
$$

which can be inserted into (3.7) to obtain

$$
b_{k}(\vartheta)=\sum_{r \text { odd }}\left(\frac{2 e^{-2 \pi i k r \alpha}}{i r \pi}\right)\left(\vartheta, \chi_{-r}\right)=\sum_{r \text { odd }}\left(\frac{4}{r^{2} \pi^{2}}\right) e^{2 \pi i k r \alpha} .
$$

Notice that since $\sum_{k=0}^{\infty}(2 k+1)^{-2}=\pi^{2} / 8$, we find

$$
b_{0} \equiv \sigma_{\vartheta}\left(S^{1}\right)=\sum_{r \text { odd }}\left(\frac{4}{r^{2} \pi^{2}}\right)=1
$$

as expected. The numbers $b_{k}$ are thus the Fourier coefficients of the spectral "density" (cf. $(3.8))$

$$
\rho(\lambda) \equiv \frac{d \sigma_{\vartheta}(\lambda)}{d \lambda}=\sum_{r \text { odd }}\left(\frac{4}{r^{2} \pi^{2}}\right) \delta(\lambda-\{r \alpha\})
$$


The coefficients (3.27) are in this case given by

$$
\sigma_{k}=\frac{4}{\pi^{2}} \sum_{\substack{r \operatorname{odd} \\ 1 /(k+1) \leq 2\|r \alpha\| \leq 1 / k}} \frac{1}{r^{2}} .
$$

Estimate (3.34) along with Corollary 3.1 then yield the following result.

Proposition 3.6. If $\alpha$ has bounded partial quotients, then

$$
\sum_{\substack{r \text { odd } \\\|r \alpha\| \leq 1 / 2 n}} \frac{1}{r^{2}}=O\left(n^{-2} \log ^{2} n\right) .
$$

If $\alpha$ is of type $\gamma \geq 1$, then for each $\epsilon>0$,

$$
\sum_{\substack{r \text { odd } \\\|r \alpha\| \leq 1 / 2 n}} \frac{1}{r^{2}}=O\left(n^{-2 / \gamma+\epsilon} \log ^{2} n\right) .
$$

If moreover $\alpha \in D C(\eta)$ with $\eta \geq 0$, then

$$
\sum_{\substack{r \text { odd } \\\|r \alpha\| \leq 1 / 2 n}} \frac{1}{r^{2}}=O\left(n^{-2 / \eta+1} \log ^{2} n\right) .
$$

We now derive a closed expression for the autocorrelation sequence $b_{k}$.

Theorem 3.7. For all $\alpha$,

$$
b_{k}(\vartheta)=1+8\{k \alpha\}(\{k \alpha\}-1)-2\{2 k \alpha\}(\{2 k \alpha\}-1) .
$$

Proof. Let $B_{n}(x)$ be the $n$th Bernoulli polynomial, defined by the generating series

$$
\sum_{n=0}^{\infty} B_{n}(x) \frac{z^{n}}{n !}=\frac{z e^{x z}}{e^{z}-1} .
$$

The first three are $B_{0}(x)=1, B_{1}(x)=x-1 / 2, B_{2}(x)=x^{2}-x+1 / 6$. Let moreover $\bar{B}_{n}(x)$ be the function which is 1-periodic and coincides with $B_{n}(x)$ in the interval $[0,1)$, that is, $\bar{B}_{n}(x)=B_{n}(\{x\})$. For $n \geq 2$ the Fourier series for $\bar{B}_{n}(x)$ is absolutely convergent and writes (see, e.g., [3, page 14])

$$
\bar{B}_{n}(x)=-\frac{n !}{(2 \pi i)^{n}} \sum_{r \neq 0} \frac{e^{2 \pi i r x}}{r^{n}} .
$$

We thus have the chain of equalities:

$$
b_{k}(\vartheta)=\frac{4}{\pi^{2}} \sum_{r \text { odd }} \frac{e^{2 \pi i k \alpha r}}{r^{2}}=\frac{4}{\pi^{2}}\left[\sum_{r \neq 0} \frac{e^{2 \pi i k \alpha r}}{r^{2}}-\frac{1}{4} \sum_{r \neq 0} \frac{e^{2 \pi i 2 k \alpha r}}{r^{2}}\right]=8 \bar{B}_{2}(k \alpha)-2 \bar{B}_{2}(2 k \alpha) .
$$

The desired formula now follows from the explicit expression of $\bar{B}_{2}(x)$. 
Now, putting together (3.9), (3.10), and (3.40) we get

$$
D S_{n}=\frac{4}{\pi^{2}} \sum_{r \text { odd }} \frac{\Phi_{n}(\{r \alpha\})}{r^{2}},
$$

and, by (3.12),

$$
\int_{0}^{1} D S_{n} d \alpha=n
$$

that is, averaging over the (normalized) set of rotation angles yields a normal dispersion. Cesàro averaging over $n$ yields instead the following asymptotic behavior.

Proposition 3.8. For all irrational $\alpha$ having an infinite subsequence of odd $q_{k}$ 's,

$$
\lim _{n \rightarrow \infty}\left\langle D S_{n}\right\rangle=\infty
$$

Proof. Let us first note that the function $\Phi_{n}(x)$ satisfies the symmetry property $\Phi_{n}(x)=$ $\Phi_{n}(1-x)$ for $0 \leq x \leq 1$, so that $\Phi_{n}(\{r \alpha\})=\Phi_{n}(\|r \alpha\|)$ where $\|a\|=\min \{|a-p|: p \in \mathbb{Z}\}$. Formula (3.14) then reads

$$
\lim _{n \rightarrow \infty}\left\langle D S_{n}\right\rangle=\frac{2}{\pi^{2}} \sum_{r \text { odd }} \frac{1}{r^{2} \sin ^{2} \pi\|r \alpha\|} .
$$

On the other hand, from (3.18) and $\|r \alpha\| \leq 1 / 2$ we deduce that

$$
\frac{1}{\pi^{2}(r \cdot\|r \alpha\|)^{2}} \leq \frac{1}{r^{2} \sin ^{2} \pi\|r \alpha\|} \leq \frac{1}{4(r \cdot\|r \alpha\|)^{2}} .
$$

For $\alpha$ irrational we have $q_{k} \cdot\left\|q_{k} \alpha\right\|=O\left(q_{k}^{1-\gamma}\right)$ where $\gamma \geq 1$ is the type of $\alpha$. Therefore under our assumption on the $q_{k}$ 's the series in (3.52) is divergent.

We conclude by showing how the different behavior of $\Phi_{n}(x)$ and $\left\langle\Phi_{n}(x)\right\rangle$ discussed in the previous subsection translates into different lower bounds for $D S_{n}$ and $\left\langle D S_{n}\right\rangle$.

Suppose that $\alpha$ is chosen so that all $q_{k}$ 's are odd. Then using (3.19) and (1.29) we have

$$
D S_{n} \geq c_{1} \sum_{\substack{r \text { odd } \\\|r \alpha\|<1 / 2 n}} \frac{n^{2}}{r^{2}} \geq c_{2} \frac{n^{2}}{q_{k_{n}}^{2}} .
$$

If $\alpha$ has bounded partial quotients, then the above estimate is not very informative in that it gives $n^{2} / q_{k_{n}}^{2} \asymp$ const. On the other hand, putting together (3.54) and Lemma 1.4 we get the following.

THEOREM 3.9. If $\alpha$ is of type $\gamma>1$ and the $q_{k}$ 's are all odd, then an infinite subsequence $\left\{n_{j}\right\}$ can be found such that for each $\epsilon>0$ there is a constant $C_{\epsilon}>0$ so that

$$
D S_{n_{j}} \geq C_{\epsilon} n_{j}^{2(1-(1 /(\gamma-\epsilon)))} \text {. }
$$


Concerning $\left\langle D S_{n}\right\rangle$, from (3.24) (or else (3.41) and (3.32)) along with (1.23) we get (assuming all the $q_{k}$ 's odd)

$$
\left\langle D S_{n}\right\rangle \geq c_{3} \sum_{\substack{r \text { odd } \\\|r \alpha\| \geq 1 / 2 n}} \frac{1}{(r \cdot\|r \alpha\|)^{2}} \geq c_{4} \sum_{k=0}^{k_{n}-1} \frac{1}{\left(q_{k} \cdot f_{k}\right)^{2}} \geq c_{4} \sum_{k=1}^{k_{n}} a_{k}^{2} .
$$

Using Lemma 1.1 we then obtain the following result.

Theorem 3.10. There is a constant $C>0$ such that

$$
q_{\ell} \leq n<q_{\ell+1} \Longrightarrow\left\langle D S_{n}\right\rangle \geq C \sum_{k=1}^{\ell}{ }^{*} a_{k}^{2}
$$

where the starred sum is restricted to those $k$ for which $q_{k-1}$ is odd.

This inequality can be used to derive lower bounds for various types of $\alpha$. For example, if $\alpha$ is of type $\gamma>1$, the last term of the sum in (3.57) can be estimated by noting that we can find an infinite subsequence of $r$ values so that $r^{\gamma} \cdot\|r \alpha\|<C_{1}$. By (1.24), this is equivalent to $q_{\ell+1}>C_{2} q_{\ell}^{\gamma}$ and $G^{\ell}(\alpha)<C_{3}\left(\prod_{k=0}^{\ell-1} G^{k}(\alpha)\right)^{\gamma-1}$, where, again, the inequalities are supposed to hold along a suitable infinite subsequence of $\ell$ values. Using (1.17) we then get for all $q_{\ell} \leq n \leq A q_{\ell}^{\gamma}$,

$$
a_{\ell} \geq \frac{1}{G^{\ell}(\alpha)}>c_{5}\left(\prod_{k=0}^{\ell-1} G^{k}(\alpha)\right)^{1-\gamma}>c_{6} q_{\ell}^{\gamma-1}>c_{7} n^{1-1 / \gamma}
$$

An interesting estimate can be easily obtained for a class of numbers $\alpha$ with bounded partial quotients. We first need the following result.

Lemma 3.11. Given any irrational $\alpha$, for every pair $q_{k}, q_{k+1}$ of consecutive denominators, at least one of them is odd.

Proof. By induction: start with $q_{0}=1$ and by the defining recursion $q_{k+1}=a_{k+1} q_{k}+q_{k-1}$ one sees that if $q_{k-1}$ is odd and $q_{k}$ is even, then $q_{k+1}$ has to be odd.

For example for the golden mean $\alpha=(\sqrt{5}-1) / 2=[1,1,1, \ldots]$ the $q_{k}$ 's are the Fibonacci numbers $1,1,2,3,5,8,13,21,34, \ldots$, and the frequency of odd $q_{k}$ 's is $2 / 3$. For $\alpha=\sqrt{2}-1=[2,2,2, \ldots]$ the $q_{k}$ 's are numbers $1,2,5,12,29,70,169, \ldots$, and the frequency of odd $q_{k}$ 's is exactly $1 / 2$.

Finally, using Theorem 3.10, Lemma 3.11 and recalling that for $\alpha$ with bounded partial quotients the integer $\ell$ in (3.57) satisfies $\ell \asymp \log n$, we readily get the following inequality.

TheOREM 3.12. For all irrational $\alpha$ with bounded partial quotients the following holds, for n large enough:

$$
\left\langle D S_{n}\right\rangle \geq \frac{1}{2} \log n
$$




\section{Acknowledgments}

The author is indebted to Mirko Degli Esposti and Claudio Bonanno for many discussions on this and related subjects. The author also wishes to thank Alejandra Gonzales for having produced a variety of pictures (not reported here) which illustrate some of the results discussed above.

\section{References}

[1] J. Aaronson and M. Keane, The visits to zero of some deterministic random walks, Proceedings of the London Mathematical Society. Third Series 44 (1982), no. 3, 535-553.

[2] P. Alessandri and V. Berthé, Three distance theorems and combinatorics on words, L'Enseignement Mathématique 44 (1998), no. 1-2, 103-132.

[3] P. Cartier, An introduction to zeta functions, From Number Theory to Physics (Les Houches, 1989) (M. Waldschmidt, P. Moussa, J.-M. Luck, and C. Itzykson, eds.), Springer, Berlin, 1992, pp. 1-63.

[4] I. P. Cornfeld, S. V. Fomin, and Ya. G. Sinaĭ, Ergodic Theory, Fundamental Principles of Mathematical Sciences, vol. 245, Springer, New York, 1982.

[5] S. De Bièvre and G. Forni, On the growth of averaged Weyl sums for rigid rotations, Studia Mathematica 130 (1998), no. 3, 199-212.

[6] K. Florek, Une remarque sur la repartition des nombres $n \xi \bmod 1$, Colloquium Mathematicum (Wroclaw) 2 (1951), 323-324.

[7] C. Godrèche, J. M. Luck, and F. Vallet, Quasiperiodicity and types of order: a study in one dimension, Journal of Physics A 20 (1987), no. 13, 4483-4499.

[8] A. G. Kachurovskii, The rate of convergence in ergodic theorems, Russian Mathematics Surveys 51 (1996), no. 4, 653-703.

[9] J.-P. Kahane, Some Random Series of Functions, 2nd ed., Cambridge Studies in Advanced Mathematics, vol. 5, Cambridge University Press, Cambridge, 1985.

[10] A. I. Khinchin, Continued Fractions, University of Chicago Press, Illinois, 1964.

[11] L. Kuipers and H. Niederreiter, Uniform Distribution of Sequences, John Wiley \& Sons, New York, 1974.

[12] A. G. Postnikov, Tauberian theory and its applications, Proceedings of the Steklov Institute of Mathematics 144 (1980), no. 2, 138.

[13] A. M. Rockett and P. Szüsz, Continued Fractions, World Scientific, New Jersey, 1992.

[14] N. B. Slater, The distribution of the integers $N$ for which $\theta N<\phi$, Proceedings of the Cambridge Philosophical Society 46 (1950), 525-534.

[15] F. E. Su, Convergence of random walks on the circle generated by an irrational rotation, Transactions of the American Mathematical Society 350 (1998), no. 9, 3717-3741.

Stefano Isola: Dipartimento di Matematica e Informatica, Università di Camerino, via Madonna delle Carceri, 62032 Camerino, Italy

E-mail address: stefano.isola@unicam.it 


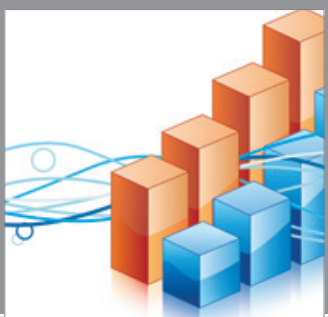

Advances in

Operations Research

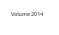

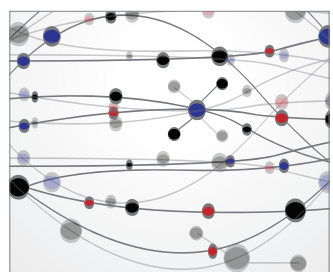

\section{The Scientific} World Journal
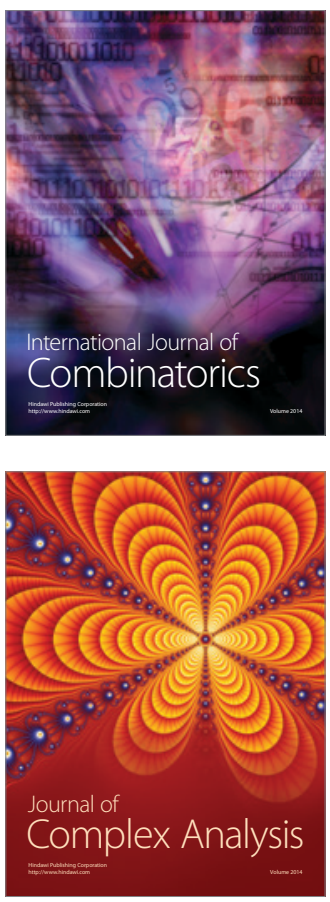

International Journal of

Mathematics and

Mathematical

Sciences
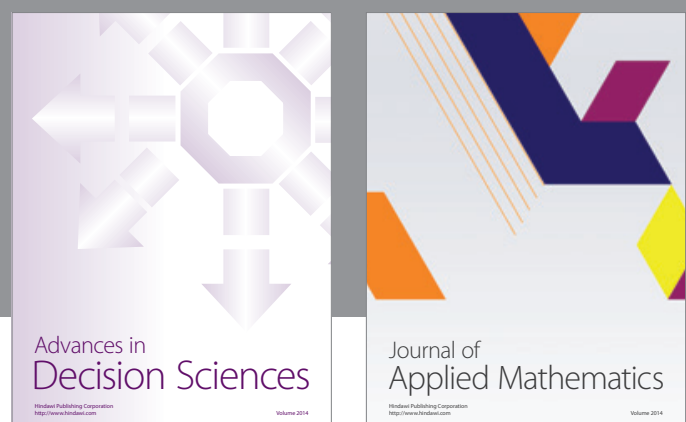

Journal of

Applied Mathematics
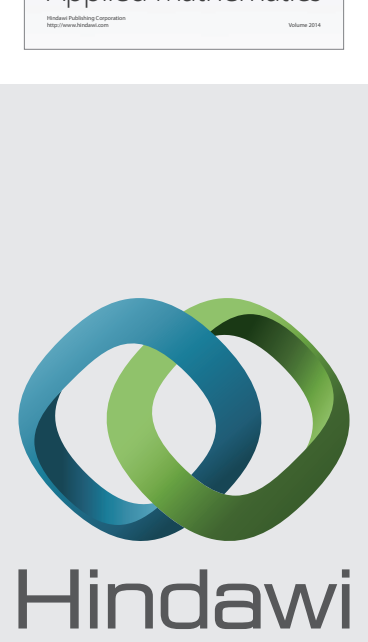

Submit your manuscripts at http://www.hindawi.com
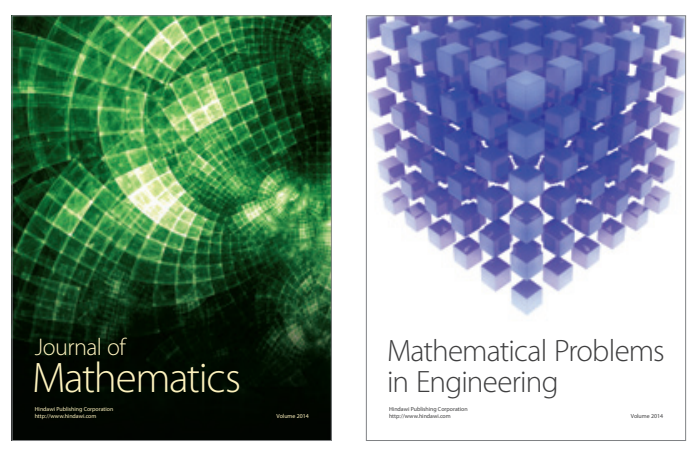

Mathematical Problems in Engineering
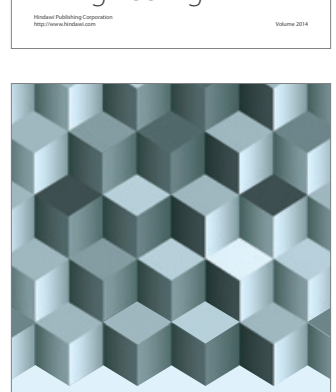

Journal of

Function Spaces
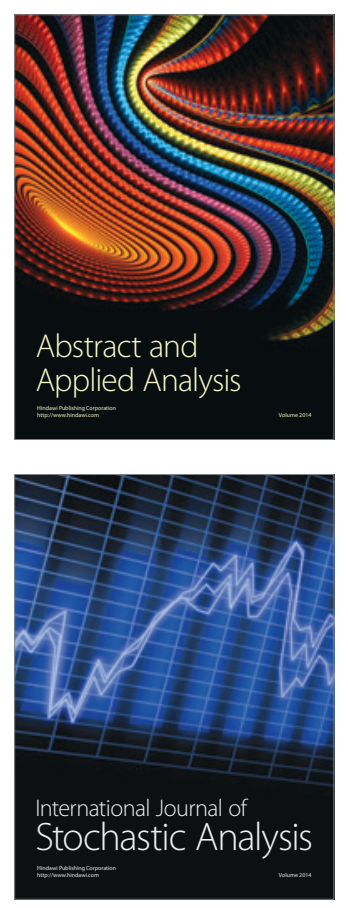

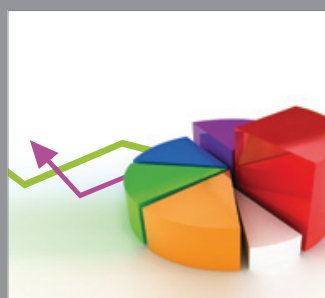

ournal of

Probability and Statistics

Promensencen
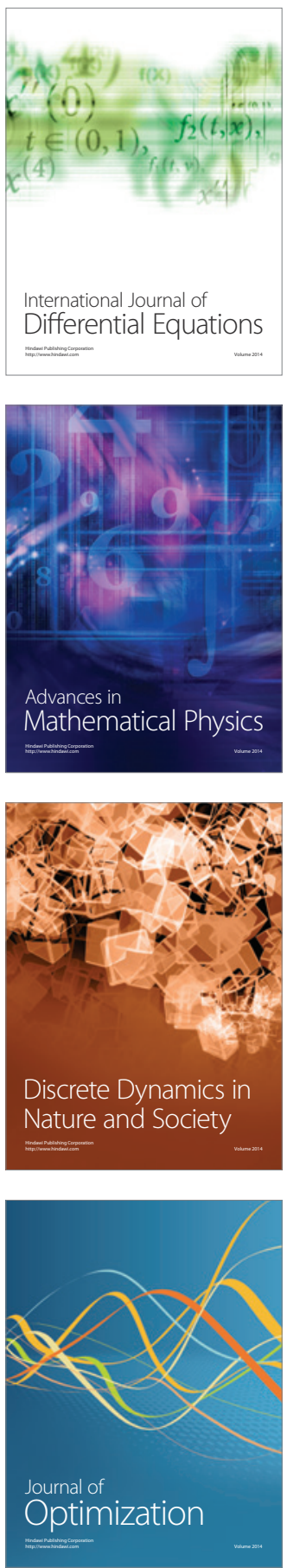